\title{
Self-Reproducing Programs in Common Lisp
}

\author{
Peter Norvig \\ Computer Science Division, University of California \\ Berkeley, CA 94720 \\ norvigateak . berkeley . edu
}

This paper reviews the classic self-reproducing expressions in Lisp, and presents some new ones that are unique to Common Lisp.

\section{The Classic Self-Evaluating Expressions}

The search for self-reproducing programs - programs that print their own sourcesis a common exercise going back at least to [Bratley and Millo, 1972]. In Lisp, there is no such notion as a program per se, so the exercise is instead to find self-reproducing or self-evaluating expressions. Of course, there are many trivial self-evaluating atoms, such as these two:

t

2

Thus, it is traditional to limit the quest to non-atomic expressions. It is wellknown that the following expression fits the bill, and as it uses only the most basic primitives, it will work in any dialect of Lisp:

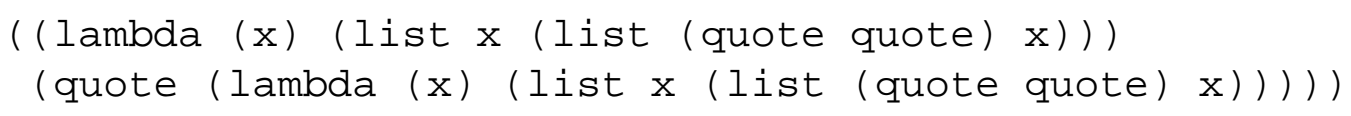

There are a few interesting variations on this theme. In a modern Lisp with backquote notation, a more succinct version is possible: 
((lambda (x) (list $\left.\left.x y^{\prime}, x\right)\right)$ ' (lambda (x) (list x '', x)))

In Common Lisp (but not Scheme) it is possible to write an equivalent but more obtuse version:

((lambda (list) (list list ', list))

' (lambda (list) (list list '', list)))

Conversely, in a Scheme where the printed representation of a function is the source code of the function, we can simply say:

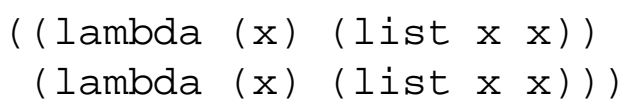

This version is also self-reproducing in Common Lisp if you install the following handy macro definition for $1 \mathrm{ambda},{ }^{1}$ and if your Common Lisp prints macro expansions as the original source code.

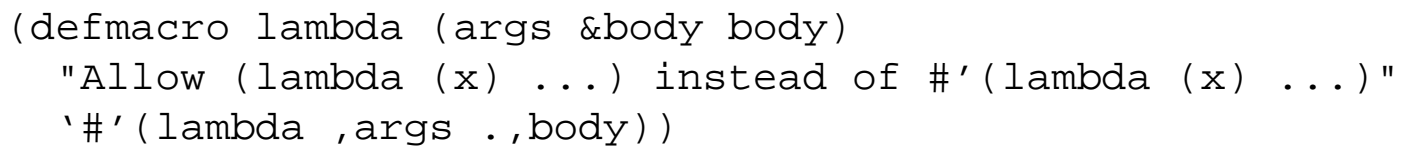

Jon L White has suggested that the self-reference implicit in these self-evaluating expressions is reminiscent of the self-reference done by the $\mathrm{Y}$ combinator. The $\mathrm{Y}$ combinator is what one needs to add to the lambda calculus to allow recursion (see [Field and Harrison, 1988, p. 133]). In a normal-order reduction calculus with Scheme syntax, we can write Y as:

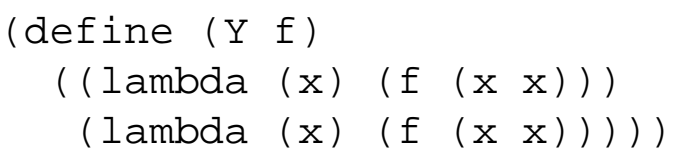

Indeed, the body of Y looks just like the canonical self-evaluation expression with list replaced by $f$, and with an extra function call thrown in. We can show that this is a proper definition of $Y$ with the following proof of the identity $(Y)=(f$ (Y f)):

\footnotetext{
${ }^{1}$ If you hate those unsightly \#' marks as much as I do, you'll use this macro even when you aren't playing with self-evaluating expressions.
} 


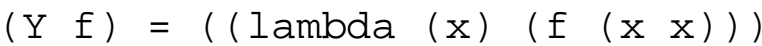

$$
\begin{aligned}
& \text { (lambda (x) (f }(x \quad x))) \text { ) } \\
& =(f((\text { lambda }(x)(f(x \quad x))) \\
& (\text { lambda }(x)(f(x \quad x))))) \\
& \left.=\left(\begin{array}{ll}
f & f
\end{array}\right)\right)
\end{aligned}
$$

The key to this derivation is that ( $\mathrm{Y}$ f) reproduces itself, along with an additional call to f. Our self-evaluating expression reproduces itself in the same way, but doesn't add an additional call. However, we can show that

$$
(Y \text { identity })=(\text { identity }(Y \text { identity }))=(Y \text { identity })
$$

So ( $Y$ identity) is self-evaluating in the normal-order reduction calculus, although in an applicative-order language like Lisp it results in infinite recursion (see [Gabriel, 1988] for a discussion of applicative Y).

\section{Some New Self-Evaluating Expressions}

Let's return to the main point of this article: novel self-evaluating expressions. Once the door is opened to the full lexical conventions of Common Lisp, some very succinct new solutions are possible. Consider:

$\# 1={ }^{\prime} \# 1 \#$

This is the list whose first element is the symbol quote and whose second element is the list itself. While the expression is certainly self-evaluating, it does have the drawback that it is only self-reproducing in an environment where *print-circle* is non-nil. That restriction is lifted with the following version:

$\# 1=($ setq $*$ print-circle* $\quad \# 1 \#)$

Those who complain that the goal should be to find a self-evaluating function call can embed these solutions in lambda expressions:

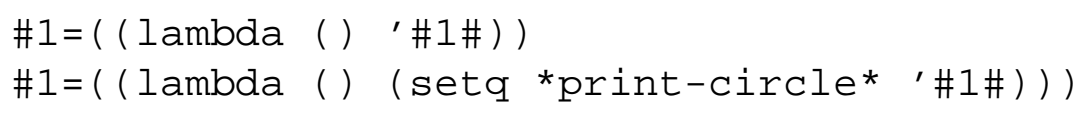


An alternate approach makes use of the oft-forgotten variable -, which in Common Lisp is bound to the current input to the read-eval-print loop (just as * is bound to the previous result). Thus, the following two expressions are selfreproducing when typed to a read-eval-print loop:

$-$

(identity -)

The first of these is the only non-constant atomic expression that is guaranteed to be self-evaluating, while the second is of course non-atomic.

As an aside, the following is one of the shortest infinite looping expression:

$($ eval -$)$

Richard Fateman provided a few more short infinite looping expression:

(loop)

$\# 1=(\operatorname{progn} \# 1 \#)$

In fact, an infinite loop results when progn is replaced by any function, or by multiple-value-call, multiple-value-progl, tagbody, assert, unwind-protect, case, prog1, prog2, do, and, or, when or unless.

In summary, it has been assumed that the best way to write a self-reproducing program is to bind a variable to part of the program, and then output that variable twice, along with enough "glue" to comprise the rest of the program. This short paper shows that it is also possible to get the same results by circular reference using the Y combinator or Common Lisp's unique \# $1=$ and - conventions.

\section{References}

[1] Bratley, P. and Millo, J. Computer Recreations. Self-reproducing automata. Software Practice and Experience, 2, (1972) 397-400.

[2] Field, A.J. and Harrison, P.G. Functional Programming, Addison-Wesley, (1988).

[3] Gabriel, R. The Why of Y. Lisp Pointers, 2 2, (1988) 15-25. 